\title{
Penile venous stripping surgery is a viable option for erectile dysfunction after unsuccessful vascular interventions
}
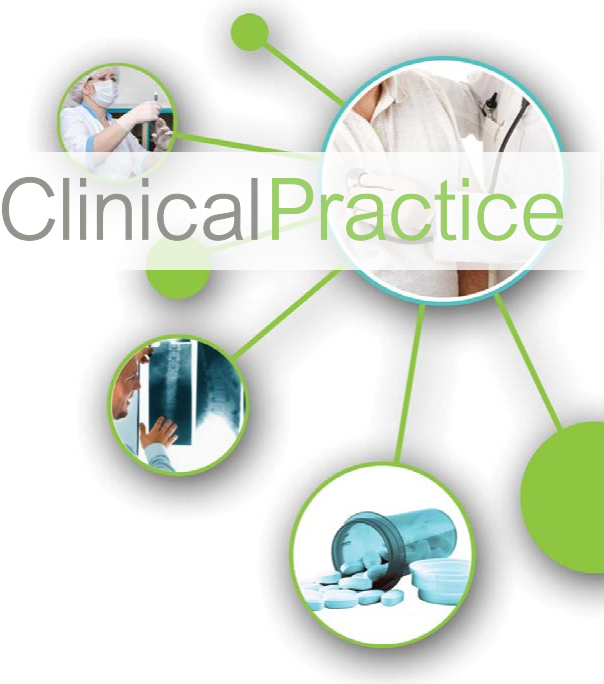

To study whether penile venous stripping can improve erectile function after unsuccessful prior vascular interventions.

Methods: This study is a retrospective review of the 49 consecutive patients that sought our assistance between 1999 and 2016 after receiving unsatisfactory vascular interventions elsewhere. Patients were evaluated by the abridged 5 -item version of the International Index of Erectile Function (IIEF-5) and pharmaco-cavernosography. After the evaluation, the 36 patients without cardiovascular problems underwent a salvaging penile venous stripping and while the 13 remaining patients did not undergo the procedure. A circumferential incision was first made to strip the erection-related veins with 6-0 nylon sutures. A median longitudinal pubic incision was used to complete the stripping proximally until the infrapubic angle was reached. Finally, the wound was closed with 5-0 chromic sutures.

Results: The follow-up period ranged between 1.0 and 16.5 years. Patients reported adequate and acceptable penile morphology postoperatively. Though there was no significant difference in IIEF- 5 scores between the two groups preoperatively ( $n=13,7.3 \pm 1.9$ vs. $n=36,7.6 \pm 2.3, p=0.11$ ), there was a statistically significant difference between treatment ( $n=36,7.6 \pm 2.1$ vs. $17.4 \pm 4.1, p<0.001$ ) and control groups ( $n=13,5.9 \pm 1.8$ vs. $n=29,18.7 \pm 3.4, p<0.001$ ). Postoperative cavernosograms confirmed that the paired corpora cavernosa are an ideal chamber for intracorporeal fluid retention in all patients, particularly, the penile crura displayed a stronger radiopaque than that of femoral cortex. Furthermore, we identified certain unexpected complications, such as embolization coils lodged in the cardiopulmonary avenues and electrocautery-induced fibrosis from prior interventions.

Conclusions: Penile venous stripping appears to be a viable option for those who have undergone unsatisfactory prior vascular interventions for erectile dysfunction.

KEYWORDS: penile vascular interventions, penile venous ligation, penile venous stripping, treatment outcome, vasculogenic impotence

\section{Abbreviations}

CC- corpora cavernosa; CV- cavernosal vein; DA- dorsal artery; DDV- deep dorsal vein; EDerectile dysfunction; IIEF - international index of erectile function; PAS- pudendal arterial stent; PAR- penile arterial reconstruction; PAVpara-arterial vein; PDE-5- phosphodiesterase type 5; PGE-E1- prostaglandin- E1; PVEpenile venous embolization; PVL- penile venous ligation; PVSS- penile venous stripping surgery; USPTO- United States Patent and Trade Office; VOD- veno-occlusive dysfunction

\section{Background}

Erectile dysfunction is an ongoing management issue and has become more prevalent in the aging population. In the human penis, erectile capability depends on the sinusoids, which are specifically distributed in the glans penis, corpus spongiosum, and corpora cavernosa (CC) independently [1]. The CC sinusoids are encircled by the bilayered tunica albuginea, whose peculiar design provides a bony rigidity during a full erection [2]. Because the CC arterial blood supply increases from $2-3 \mathrm{ml} /$ $\mathrm{min}$ in flaccid to $60-80 \mathrm{ml} / \mathrm{min}$ at full erection states, healthy erectile function relies on a fully functioning erection-related vascular system [3]. That is, a healthy cardiovascular system is a prerequisite for meaningful erectile function. Failure to attain or maintain a rigid CC —erectile dysfunction (ED) - is a result of either penile arterial insufficiency or penile veno-occlusive dysfunction (VOD). Consequently, various vascular interventions have been used to either increase CC arterial supply or reduce venous drainage from the CC
Cheng-Hsing Hsieh ${ }^{1}$, Cheng-Wen Chen ${ }^{2}$, HungMeng Huang ${ }^{2}$, Hollis Johnson ${ }^{3}$, Ru-Jeng Teng ${ }^{3}$ and Geng-Long $\mathrm{Hsu}^{4 *}$

${ }^{1}$ Department of Urology, Taipei Tzuchi Hospital, School of Medicine, Buddhist Tzu Chi University, Hualien, Taiwan

${ }^{2}$ Department of Urology, Taipei City Heping Fuyou Branch Hospital, Taipei, Taiwan

${ }^{3}$ Department of Pediatrics, Medical College of Wisconsin, Milwaukee, Wisconsin, USA

${ }^{4}$ Microsurgical Potency Reconstruction and Research Center, Hsu's Andrology and National Taiwan University, Taipei, Taiwan

*Author for correspondence:

genglonghsu@gmail.com;glhsu@ ha.mc.ntu.edu.tw 
sinusoids [4]. The former includes vasoactive agents (such as phosphodiesterase type 5 (PDE5) inhibitor and prostaglandin-E1 (PGE-E1)), penile arterial reconstruction (PAR) [5] and pudendal arterial stents (PAS) [6], whereas the latter includes penile venous ligation (PVL) [7], penile venous stripping surgery (PVSS) [8], penile venous sclerotherapy [9] and penile venous embolization (PVE) [10]. Deep dorsal vein (DDV) arterialization, a hybrid treatment, was commonly used previous to more recent advancements [11]. Every treatment modality must be somewhat effective; otherwise, repeated experiments would not be performed on the human body. This is true with all vascular interventions, particularly to the PVL, which involves an incomplete excision of offensive veins [12].

In 1873, Parona proposed that the penile dorsal vein can be dysfunctional due to varicosity [13]. In 1902, Wooten proposed ligation of the deep penile vein as a cure for atonic impotence [14]. Although Lowsley and Rueda had performed this procedure in many cases before 1953 [15], similar surgeries for restoring erectile function were not popular until 1985 [16]. However, its popularity dwindled over the course of the next decade [17]. There are understandable reasons to abandon PVL because of disappointing outcomes and adverse effects, such as irreversible deformity and permanent penile numbness [18]. Despite these drawbacks, our PVSS was developed with our advanced understanding of the penile tunical and venous anatomy associated with the erection mechanism beginning with research as far back as 1986 [19]. After successfully implementing our refined PVSS technique in a large number of patients, we have encountered international patients seeking this due to their poor functional outcomes or unexpected adverse effects after previous penile vascular interventions. Although we recommend PVSS only to patients who are non-responders to phosphodiesterase-5 (PDE-5) inhibitors, we cannot decline patients that respond to PED5 inhibitors [20]. Herein, we report the results of salvaging PVSS in 36 consecutive patients who had prior vascular intervention.

\section{Methods}

\section{- Patient population}

Between July 1999 and March 2016, 49 consecutive patients sought our assistance because of refractory ED after they had undergone prior penile vascular interventions 1 to 8 years earlier elsewhere. Patients were excluded for savaging PVSS if they had either an uncontrolled cardiovascular problem or suspected penile arterial misligation in prior surgery. The abridged 5-item version of the International Index of Erectile Function (IIEF-5) and pharmaco-cavernosography were used to evaluate the outcomes. Of them 36 men underwent a salvaging penile venous stripping; 11 presented with penile deformity and the remaining 13 patients did not receive the surgery. The PVSS was developed with consideration to of our advanced penile anatomy research and clinical study (FIGURE 1A-B), thus resulting in an improved procedure for restoring erectile function. As a clinical application of approved institutional review boards of China Medical University and Taipei Tzuchi Hospital, all patients provided written informed consent and were documented to have persistent VOD according to our dual pharmaco-cavernosography. The first set of cavernosograms (FIGURE 1C-D) were obtained immediately after an intracavernous injection of a $60-\mathrm{ml}$ solution prepared from iohexol $(25 \mathrm{ml}$; Omnipaque, GE Healthcare, Cork, Ireland) and normal saline $(35 \mathrm{ml})$ in a $60 \mathrm{ml}$ syringe connected to a 19-gauge scalp vein needle. The second set of pharmacocavernosograms (FIGURE 1E) was obtained 30 minutes after a $20-\mu \mathrm{g}$ injection of prostaglandin E1 (PGE1) performed using the same line. Meanwhile, arterial function was assessed using the PGE1 test and Doppler sonography. Another set of cavernosograms (FIGURE 1F,G,H) were routinely undertaken for comparison immediately after operation where the radiopacity of penile crura was meticulously compared to that of the femoral cortex. Twenty nine of the 42 patients underwent PVL earlier, 3 underwent PVE (FIGURE 2A-B), 2 received PAS (FIGURE 2C,D,E,F) and one each underwent PAR (FIGURE 3) and venous sclerotherapy, respectively. PVSS is superior to PVL (incomplete venous removal included) in terms of erectile function restoration, with no recurrent venous leakage as reported in the postoperative follow-up imaging study [12]. Our salvaging procedure included a careful relief of fibrotic tissues, if extant, followed by PVSS under a peak head loupe magnification. The venous stumps were then ligated using 6-0 nylon as close to the tunica albuginea as possible. The number of ligation sites was as high as 132 . The surgery was performed on 
A

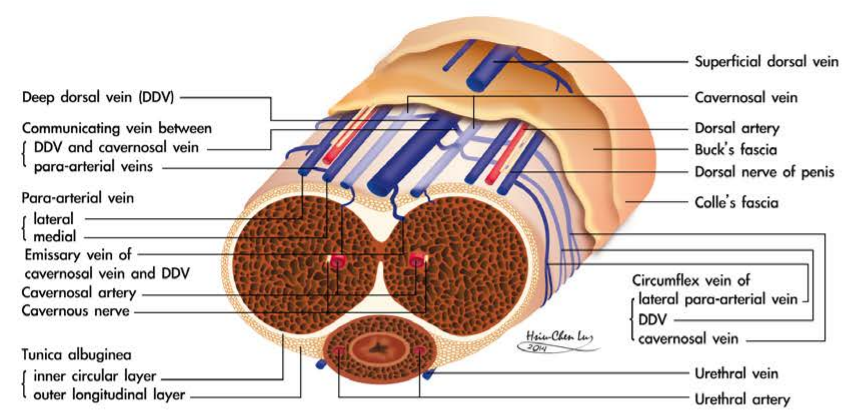

B

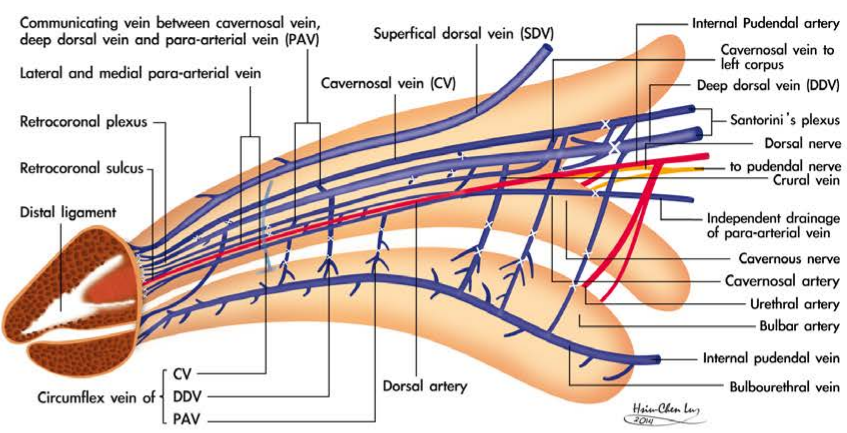

Hainclentan
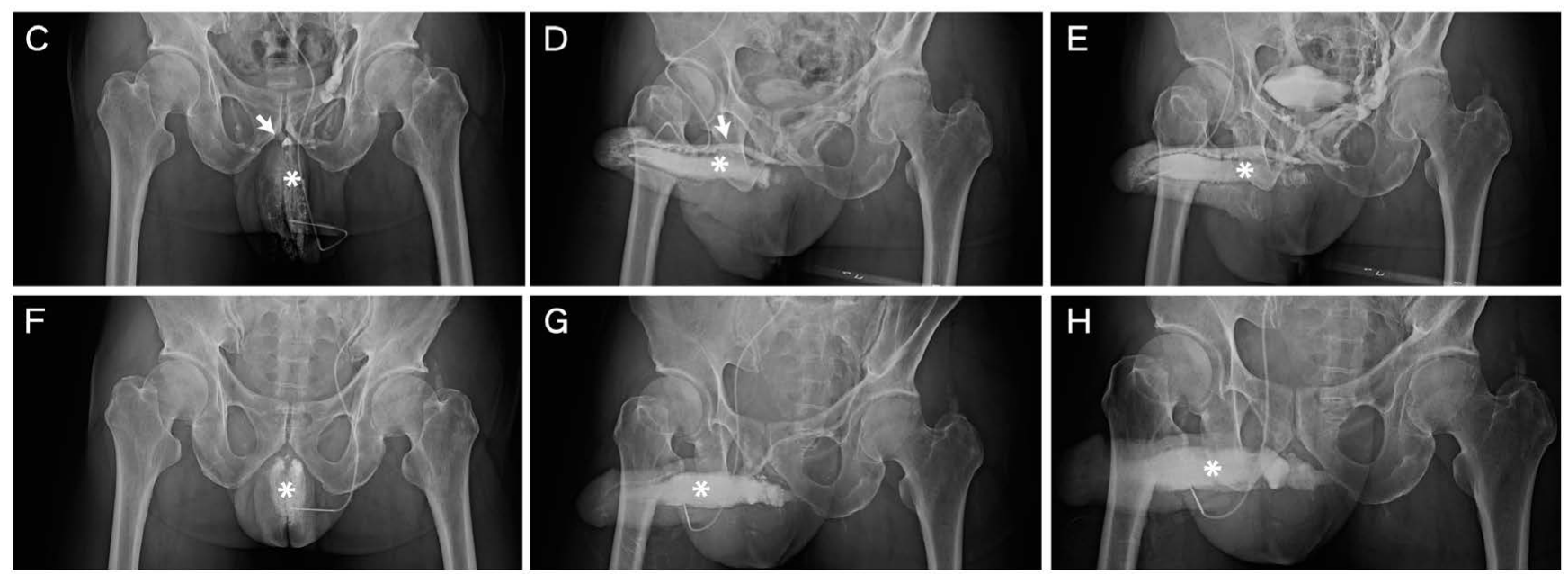

Figure 1: Schematic drawings of the advanced penile venous anatomy and imaging of dual pharmaco-cavernosograpy in this study. (A) Cross section of the mid-portion in human penis. The erection-related veins are dipicted in an imaginary arc on the dorsal aspect of the tunica albuginea. The deep dorsal vein (DDV) is flanked by a pair of cavernosal veins (CVs), whereas each dorsal artery is sandwiched by its corresponding medial and lateral para-arterial veins (PAVs). Each DDV, CV, and PAV has separate circumflex veins. (B) Upper lateral view: The DDV, consistently in the median position, receives the blood from the emissary veins in the corpora cavernosa (CC) and from the circumflex vein in the corpus spongiosum. The CVs is specific to its corresponding corpus although they lie deep. The internal pudendal artery branches to the bulbourethral artery and subsequently to the cavernosal artery, which is the major supplier to the CC, as well as the dorsal artery, which supplies blood to the glans penis. (C) The first cavernosogram (anterior-posterior (AP) view) was obtained while a 10-ml diluted iohexol solution was intracavernously injected, disclosing CC (asterisk) venous dranage to the pre-postatic plexus (arrow). (D) A $30^{\circ}$ oblique-view cavernosogram after injecting another 10-ml of contrast solution. Note the relationship between the entire venous system (arrow) and CC (asterisk) compared to that in panel B. (E) A second set of pharmacocavernosograms was obtained 20-30 minutes after $20 \mu \mathrm{g}$ of prostaglandin E1 (test) was intracavernously injected. The veins were opacified and a rigid erection was attained. (F) This cavernosogram was obtained under the same condition as that in panel $C_{;}$the offensive veins were completely stripped off. (G) This film was obtained under the same condition as that in panel D; again, a complete stripping was demonstrated. (H) Further cavernosogram confirmed the intracorporal retention of injected fluid, implying sufficient stripping of leakage veins.

an ambulatory basis under an acupuncture assisted local anaesthesia (LA) with intravenous sedation if necessary. Neither a Bovie nor a suction apparatus was used. Patients were evaluated based on their IIEF-5 scores 1.0 to 16.5 years after the surgery, and postoperative cavernosography was routinely conducted.

\section{- Penile venous stripping surgery}

Postoperative fibrosis was meticulously relieved in patients who received PVL. All patients underwent a routine PVSS procedure based on the advanced understanding of the erection- related venous anatomy (FIGURE 1A-B), where the deep dorsal vein (DDV), the pair of cavernosal veins (CVs), and the 2 pairs of paraarterial veins (PAVs) underwent operation. A circumferential incision was first made to access the DDV and a 4.0-cm longitudinal pubic incision was marked (FIGURE 4A). A milking manoeuvre, mimicking a squeeze applied to a balloon, was applied to engorge both the DDV and the CVs of the corpora cavernosa (FIGURE 4B). Then $2 \mathrm{~cm}$ of the DDV was separated from surrounding tissue and cut using scissors 1.5 $\mathrm{cm}$ proximal to coronal sulcus. With the distal 

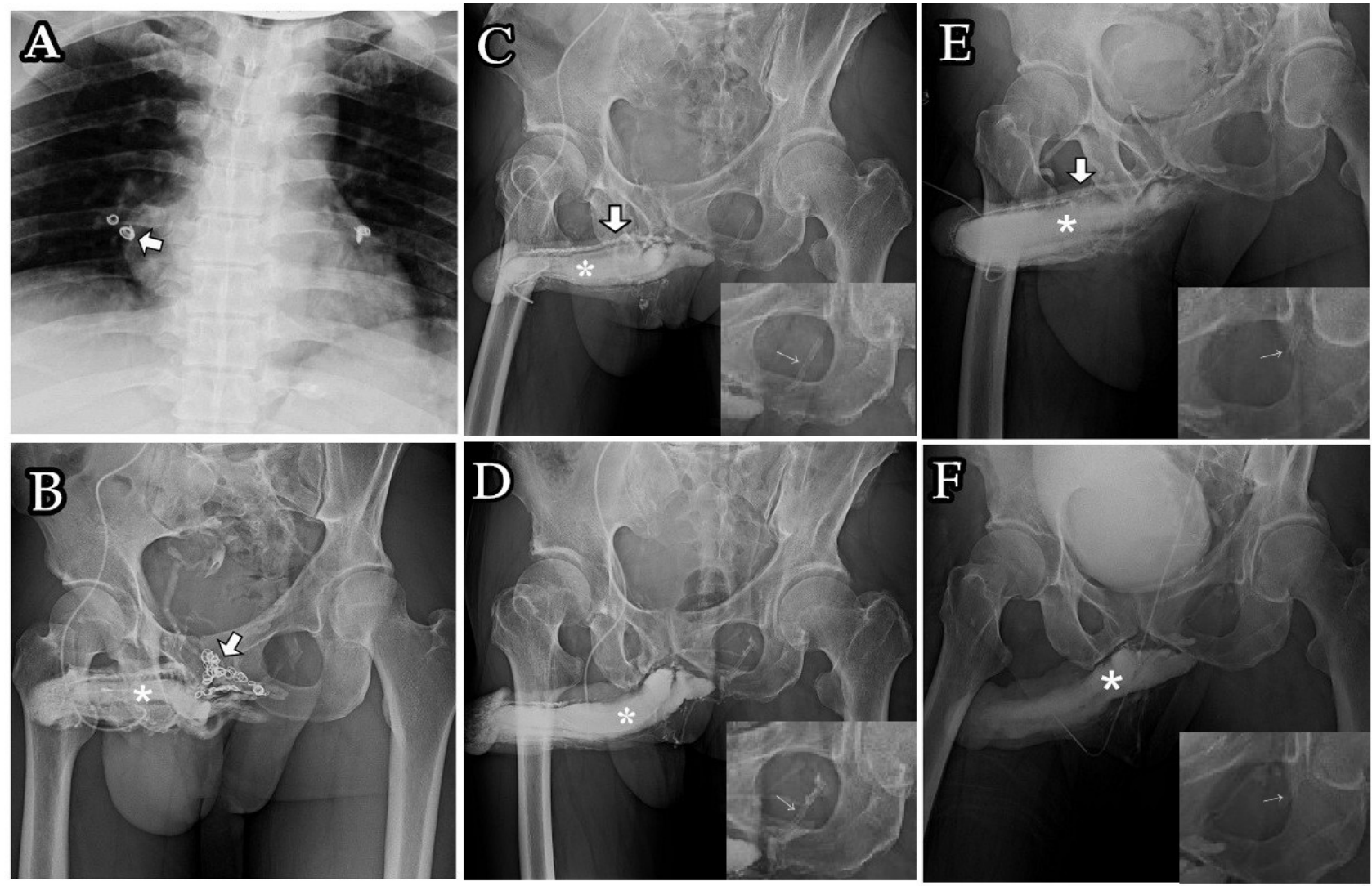

Figure 2: Imaging of patients who received prior penile venous embolization and pudendal arterial stent. (A) Several embolization coils (arrow) are lodged in the pulmonary artery and later confirmed by contrast CT scan (not shown) (B) A cavernosogram of another patient showing the many coils lodged in the Santorini plexus (arrow). (C) This cavernosogram (anterior-posterior view) was obtained while a 10-ml diluted iohexol solution was intracavernously injected. Note the immediate filling of the preprostatic veins (arrows) from the corpora cavernosa (asterisk) and the pudendal stents (arrow heads). (D) This film was obtained after penile venous stripping under the same condition as that in panel C; a complete stripping was confirmed. (E) A pharmaco-cavernosogram was undertaken and leakage veins (arrow) were present however a rigid erection was still achieved. (F) No residual veins were observed (without prostaglandin E1 injection). Note the pudendal stents (arrowheads in panel C, D, E, F) were stationary and the corpora cavernosa (asterisk) was ideal for fluid retention.

trunk serving as a guide, antegrade stripping was performed distally with the number of branches as high as 29. A pull-through manoeuvre was then continued proximally after each emissary vein was ligated closest to the outer tunica albuginea (FIGURE 4C). Subsequently, dozens of circumflex veins were markedly engorged and carefully freed after a 3-0 silk suture was fixed at the 5 or 7 o'clock position of the tunica albuginea. It is vital that a superficial dorsal vein is spared to preserve physiological circulation. Following the stripping of the distal portion of the DDV and the CVs, a longitudinal pubic incision was performed to relay the stripping procedures proximally (FIGURE 4D). Similarly, the proximal DDV and deeper-seated CVs were managed with an $85^{\circ}$ haemostat using 6-0 nylon ligatures. As a rule, 15 branches were frequently encountered within the infra-pubic angle (FIGURE 4E). Although there were 8 patients with partial resection of the DDV system, all erection- related veins remained untouched in most patients $(n=21)$. Accordingly, all residual veins were stripped thoroughly with 6-0 nylon. The deeper-seated CVs were much more prominent after the DDVs were thoroughly stripped within a 20 minute time frame. Then, the PAVs, with their visibility enhanced by squeezing the sinusoids, were segmentally ligated rather than stripped because of their fragility and proximity to the dorsal artery and dorsal nerves. Finally, the bilateral crural veins were ligated wherever possible. The wound was fashioned layer by layer up to the skin layer using 5-0 chromic sutures while an assistant consistently stretched the penile shaft (FIGURE 4F). Along with preoperative imaging (FIGURE 1C,D,E; FIGURE 2C,E; FIGURE 5A,B,C: before PVL elsewhere and FIGURE 5D,E,F: PVL elsewhere one year before our PVSS), postoperative cavernosography (FIGURE 1F,G,H; FIGURE 2D,F AND FIGURE 5G,H,I) was routinely performed to confirm the adequacy of venous 

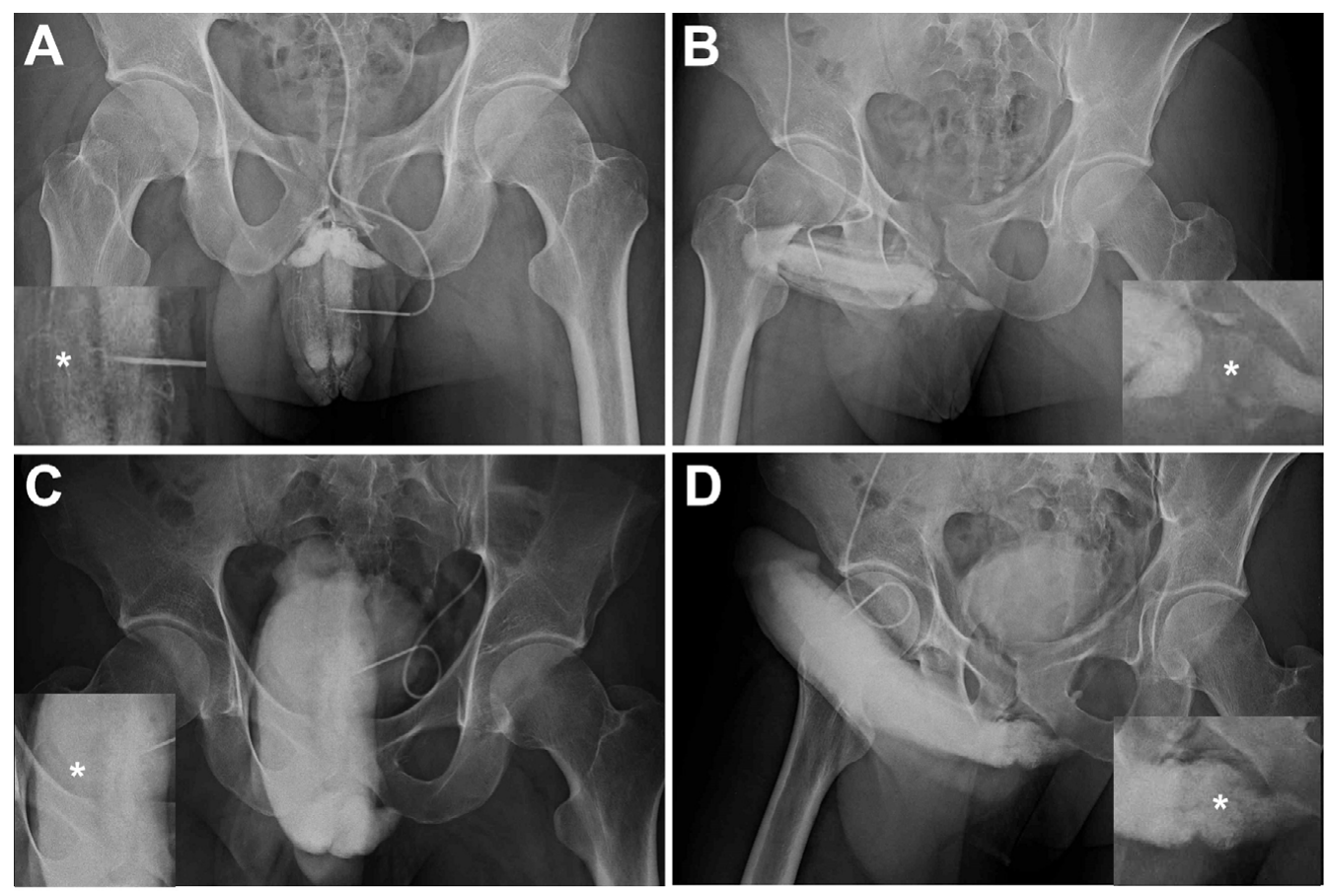

Figure 3: Cavernosograms of a 26-year-old patient who underwent prior artetrial reconstruction. (A) An anterior-posterior-view cavernosogram was obtained while a $10-\mathrm{ml}$ diluted iohexol solution was intracavernouslyinjected. The preprostatic plexus was rapidly filled and the right corpus cavernosum (asterisk) was relatively low in radiopacity, indicating an intracavernous fibrosis. (B) The radiolucent area (asterisk) was shown in this $30^{\circ}$ oblique-view cavernosogram despite further injection of the 10-ml solution. (C) The radiolucent area (asterisk) was still visible in a pharmacocavernosogram obtained 20 minutes after $20 \mu \mathrm{g}$ of prostaglandin E1 (test) was intracavernously injected. (D) Again, the radiolucent area (asterisk) was visible in a $30^{\circ}$ oblique-view pharmacocavernosogram, although it seemed to have attenuated.
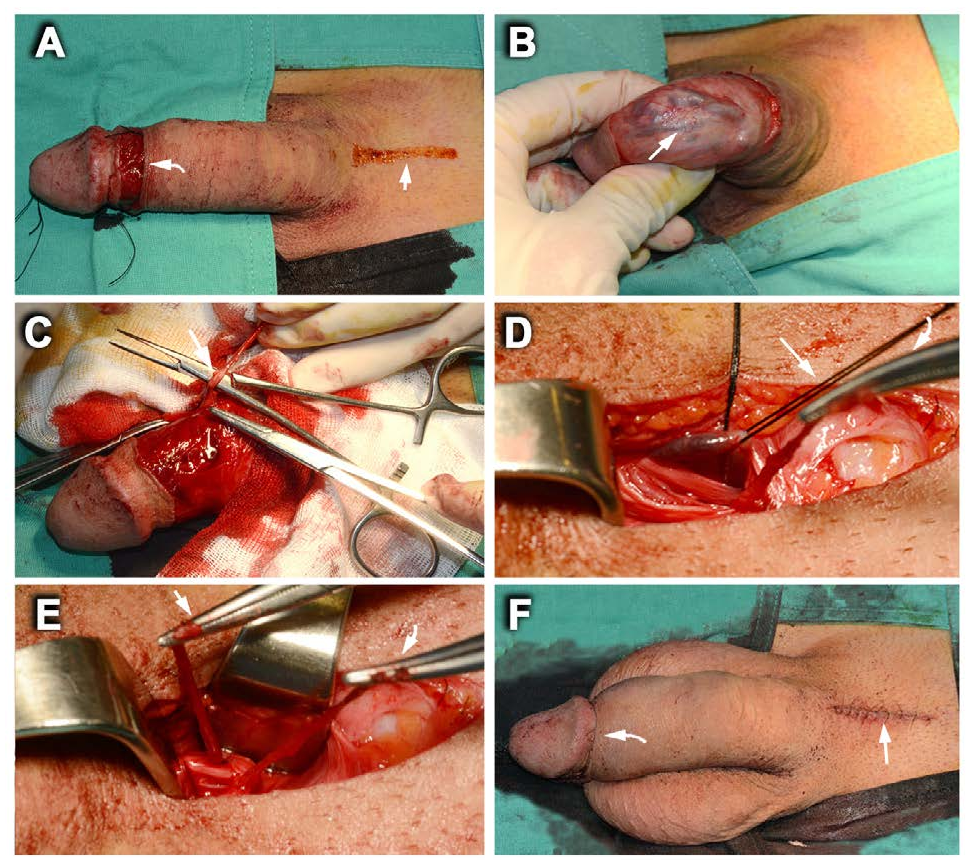

Figure 3: Photographs of penile venous stripping surgery. (A) A circumcision (curve arrow) was first performed to acess the offensive veins and an ongoing pubic longitudinal wound was marked (arrow). (B) The offensive veins (arrow) were enhanced by employing a milking manipulation of the corpora cavernosa. (C) The deep dosal vein (DDV) trunk was freed for $2 \mathrm{~cm}$, firmly held by a pair of hemostats, and cut in the middle, susequently each emissary vein (arrow) was ligated closest to the outer layer of the tunica albuginea. Venous stripping was administrated step by step using a pull-through maneuver till the penile base. (D) A longitudinal pubic incision was performed to relay the stripping precedures. The DDV stump (curve arrow) was then passed underneath and served as a guide for further stripping. Likewise, the trunk of cavernosal vein (CV) was managed (arrow). (E) An antegrade stripping was performed in a pull-through. 

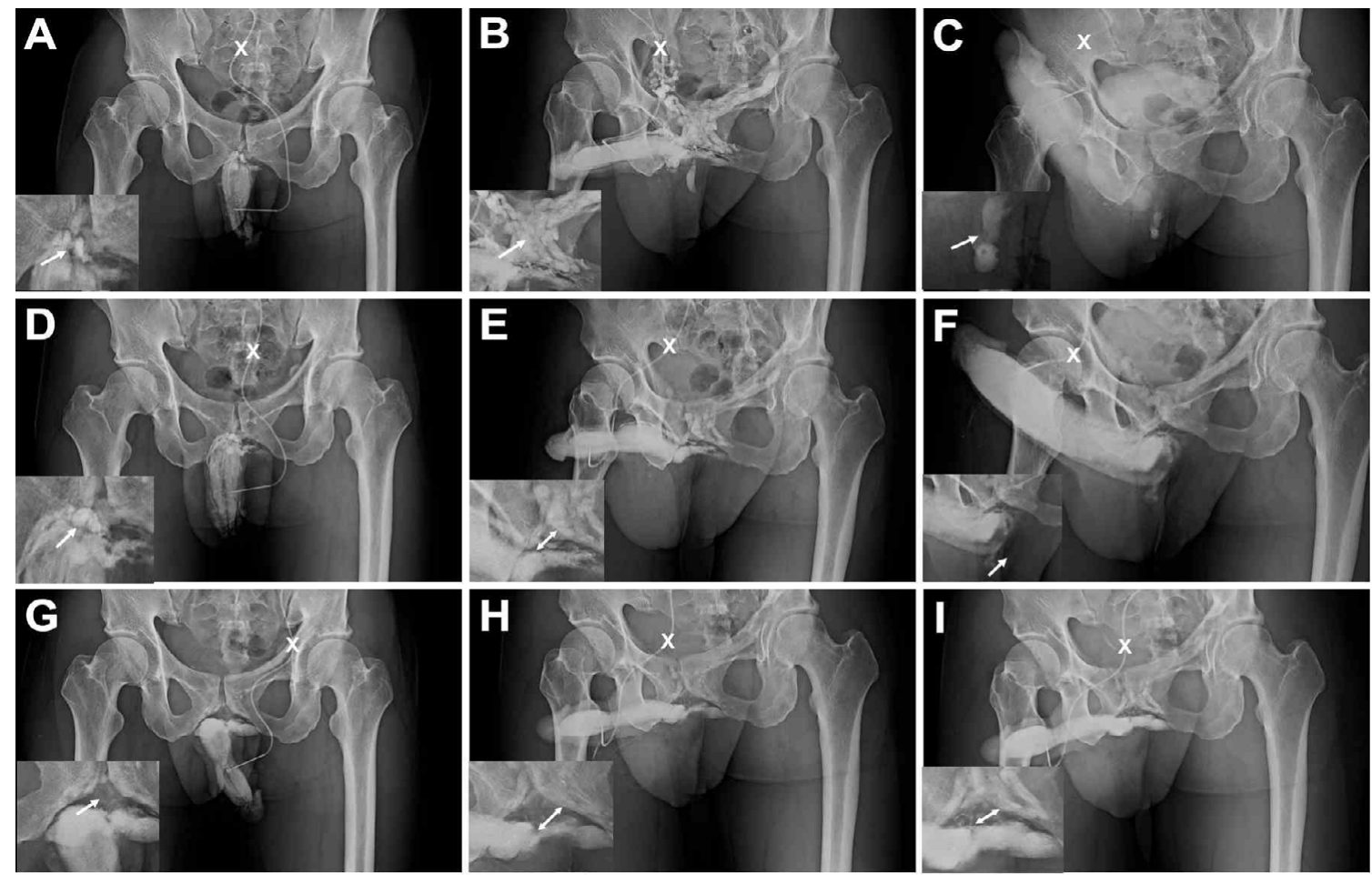

Figure 5: Cavernosograms of a 35-year-old patient who underwent prior penile venous ligation (PVL). (A) The first cavernosogram (anterior-posterior view) was obtained while a 10-ml diluted iohexol solution was intracavernously injected. The preprostatic plexus showed immediately (arrow). (B) A $30^{\circ}$ oblique-view cavernosogram was obtained after further injection of the 10-ml solution when the preprostatic venous plexus was extraordinarily large. Venous bulking was pronounced at the penile hilum (arrow). (C) This pharmacocavernosogram shows minimal leakage veins. (D) This cavernosogram shows marked residual veins (arrow) despite prior PVL. Note the differences between panels D and A. (E) This oblique cavernosogram was obtained for comparison with panel B. The preprostatic plexus (arrow) became smaller because of prior PVL. (F) An oblique view of the pharmacocavernosogram shows leakage veins, which were pronounced even though the intracavernous fluid retention appeared weak. (G) No leakage veins could be observed after PVSS. The completeness of the procedure was confirmed by a late-phase cavernosogram (H) and the characteristic intracorporal retention of injection fluid (I). We caution against using prostaglandin E1 for intracavernous injections in patients who have received PVSS.

stripping, followed by compression dressing to encircle the penile shaft. The patients were evaluated every 6 months through IIEF-5 scores and subjective grading of erection quality.

\section{- Statistical analysis}

Data with continuous values are expressed as the mean \pm standard deviation (SD). The Student's $t$ test and paired $t$ test were applied after logarithmic transformation. $\mathrm{P}<0.05$ was considered statistically significant.

\section{- Results}

The total operation time ranged between 4.5 and 8.5 hours, and the follow-up period ranged from 1.0 to 16.5 years. (TABLE 1) lists the demographics of all 49 patients noting that 36 patients underwent salvaging PVSS after prior unsuccessful penile vascular intervention. Most patients $(n=42)$ received PVL although methods varied between PVE $(n=3)$, PAS $(\mathrm{n}=2)$, PAR $(\mathrm{n}=1)$, and sclerotherapy $(\mathrm{n}=1)$. The booster injection of local anaesthetic was unexceptionally used, specifically twice in 22 patients and three times in the remaining 14. In the 29 patients who underwent prior PVL, marked fibrosis made local infiltration of lidocaine extremely difficult; despite this, successful administration was eventually achieved. In addition, electrocautery fibrosis was observed in patients who received either PAR (FIGURE 4) or PVL, whereas fibrotic features were not remarkable in those who received PAS or PVE. All patients reported satisfactory postoperative penile morphology. For erectile function, there was no difference in IIEF-5 scoring between the two groups preoperatively $(\mathrm{n}=13,7.3 \pm 1.9$ vs. $\mathrm{n}=36,7.6 \pm 2.1, \mathrm{p}=0.11)$, however, there were significant differences postoperatively both between treatments $(\mathrm{n}=36,7.6 \pm 2.1$ vs. $17.4 \pm 4.1, \mathrm{p}<0.001)$ and groups $(n=13,5.9 \pm 1.8$ vs. $n=29,17.7 \pm 3.4$, $\mathrm{p}<0.001$ ). In addition, the subjective grading of postoperative erection quality was significantly 
Table 1. Summary of the 36 patients received salvage penile venous stripping surgery (PVSS) after unsuccessful vascular interventions (VI) elsewhere since 1999.

\begin{tabular}{|c|c|c|c|c|c|c|c|c|c|}
\hline \multicolumn{3}{|c|}{ Patient } & \multicolumn{4}{|c|}{ IIEF-5 score } & \multicolumn{3}{|c|}{ IIEF-5 score } \\
\hline Prior VI & No. & Age (year) & Initial ED & Pre-op & post-op (6M) & Post-op (1.5Y) & PDE5 $\mathrm{i}^{\mathrm{a}}$ & $\mathrm{ICl}^{\mathrm{b}}$ & Implant \\
\hline \multicolumn{10}{|l|}{$P V L^{c}$} \\
\hline NPVSS & 13 & $25-61(43.3 \pm 10.9)$ & $9.2 \pm 3.9$ & $7.3 \pm 1.9$ & $6.9 \pm 1.8$ & $5.9 \pm 1.8$ & 8 & 2 & 2 \\
\hline PVSS & 29 & $26-66(40.4 \pm 11.3)$ & $9.5 \pm 2.2$ & $7.6 \pm 2.3$ & $14.9 \pm 4.3$ & $18.7 \pm 3.4$ & 7 & 2 & 2 \\
\hline PVE & 3 & $33,42,31$ & $2,8,11$ & $2,13,11$ & $2,17,18$ & 13,17 & 1 & 0 & 0 \\
\hline PAS & 2 & 67,54 & 7,5 & 7,5 & 19,5 & 25,5 & 1 & 0 & 0 \\
\hline PAR & 1 & 26 & 10 & 10 & 16 & 19 & 0 & 0 & 0 \\
\hline PVS & 1 & 24 & 10 & 10 & $17 ?$ & $?$ & & & \\
\hline Total & 49 & $41.7 \pm 11.1$ & $8.5 \pm 2.9$ & $7.6 \pm 2.1^{f}$ & $14.4 \pm 4.4^{f}$ & $17.4 \pm 4.1^{f}$ & 17 & 4 & 10 \\
\hline$p$-value ${ }^{d}$ & $N A^{e}$ & NA & NA & $>0.11$ & & $<0.001^{g}$ & $\mathrm{NA}$ & NA & $<0.001^{g}$ \\
\hline
\end{tabular}

a PDE5i indicates the abbreviation of phosphodiesterase type 5 inhibitor.

b $\mathrm{ICl}$ denotes intracavernosal injection of the prostaglandin-E1.

c PVL, PVE, PAS, PAR, PVS, PVSS and NPVSS are abbreviation of penile venous ligation, penile venous embolization, penile arterial reconstruction, pudendal arterial stent, penile venous sclerotherapy, penile venous stripping surgery and no penile venous stripping surgery respectively.

d Statistically paired t test was used in those whose post-operative period was longer than 6 months.

e NA stands not applicable.

f Data are calculated from those who underwent PVSS $(n=33)$.

9 Data are calculated from those who underwent PVL.

improved ( $\mathrm{n}=36 ; 33.8 \%$ vs. $77.6 \%, \mathrm{P}<0.02)$. Only 7 patients, all of which received surgery more than 5 years prior $(n=20)$ had IIEF5 scores of 24 or 25 , suggesting exceptional postoperative improvement. Eventually, 9 patients needed occasional help by PDE-5 inhibitors, and 2 depended on it; another 2 patients depended on intracavernous injection (ICI) therapy, whereas 1 declined any assistance after 2 attempts with partial response. Four patients, as compared to 6 in the no PVSS group, underwent penile implantation eventually. Postoperative cavernosograms confirmed that the CCs are an ideal chamber for intracorporeal fluid retention in all patients, particularly, the penile crura displayed a stronger radiopaque than that of femoral cortex (FIGURE 1F,G,H; FIGURE 2D,F AND FIGURE 5I). Although an iatrogenic trauma was microscopically repaired, arterial trauma resulting from severe fibrosis occurred in 3 of the 29 patients who underwent prior PVL. A chest X-ray revealed an unexpected pulmonary artery lodging of the embolization coils (FIGURE 2A) in a patient who underwent PVE a decade ago.

\section{Discussion}

Despite substantial advances in medicine and surgery there remains no risk-free treatment for ED. Although the introduction of PDE5 inhibitors was a great stride in impotence treatment [21], it remains an unsolved problem, leaving penile prosthesis implantation as the ultimate resort. This is reflected in the rising number of men undergoing penile implantation; this number increased from 17,540 in 2000 to 22,420 in 2009 in the United States alone [22].

ED is commonly associated with cardiovascular diseases. The prevalence of ED in patients with general vascular disease is even higher in men over 40 years of age [23]. A healthy cardiovascular system is a prerequisite for a successful erection while cardiovascular risk is especially common in the geriatric population [24]. Furthermore, extensive studies have reported that a vasogenic factor is the major cause of organic ED [25]. This justifies reducing the $\mathrm{CC}$ drainage in order to establish a milieu for an effective erection. This logic is supported by Pascal's law which depicts that pressure applied to any part of the enclosed fluid at rest is transmitted undiminished to every portion of the fluid and to the walls of the containing vessel [26]. Unsurprisingly, we have encountered patients who underwent PAR, PAS, DDV arterialization, PVL, PVE, and at the end still required penile implantation. In this report, 42 patients underwent prior PVL, 3 patients underwent PVE, 2 males received PAS, and 1 patient underwent PAR or sclerotherapy elsewhere; they then sought our assistance in PVSS at the expense of a long trip, implies that ED is a critical problem worldwide. Although various treatment modalities have been proposed to restore erectile function, certain methods (e.g. ICI and PVE) may not achieve a sustained result despite their minimally invasive 
nature. In this series, 2 patients each had received ICI and PVE. Those who received ICI complained of annoying penile deformity after several courses of ICI therapy, and unexpected migration of embolization coils was noted in one of our patients. Although those surprising complications are not immediately fatal, they can potentially compromise cardiovascular health. These side effects imply that ICI therapy can cause penile dysmorphology, and that PVE can cause cardiopulmonary insult and possibly visceral perforation. Furthermore, low-density cavernosograms were observed in most patients who had prior PAR or PVL implying the sinusoidal fibrosis is likely induced by electrocautery. Electrocautery use can be hazardous in erection restoration surgeries $[27,28]$. Sinusoidal fibrosis was observed in the penile hilum and penile shaft after PVL and PAR, respectively. This suggests that neither a Bovie nor a suction apparatus is a sound instrument for penile vascular reconstructive surgery [29].

It is extremely rare in medical history that a surgical procedure, such as the venous surgery for $\mathrm{ED}$ in this report, sustains a protracted dispute for over a century. In 1996, a 28-yearold patient from abroad was successfully treated by our PVSS procedure after suffering a decade long with ED. He attempted relentlessly to secure a surgeon in his country that would be willing to perform PVL to resolve his problem without any success. Our hemodynamic studies on cadavers were inspired by his quest so that we can differentiate ED of psychogenic origin from venogenic origin [30]. Our continuous clinical endeavour has demonstrated that penile venous surgery can benefit ED patients. PVSS is effective in cadavers, without other confounding problems (eg. psychological, hormonal, arterial, neurological, or drug-related adverse effects), and with no concern about systemic diseases and sinusoidal fibrosis, it is the ideal model to prove our concept. We are encouraged to expand our understanding and stripping technique when patients approach us after unsatisfactory functional outcomes from prior penile vascular interventions. Unfortunately, 7 out of 20 (35\%) patients whose PVSS was performed longer than 5 years after prior surgery reached IIEF- 5 of 23 or more, which was inferior to those without previous interventions [9]. Full tissue recovery seemingly requires a longer time, as suggested by the difference in IIEF-5 score between 6 months and 18 months postoperatively. Seeking a solution for ED appears to be an endless pursuit that encourages further research [31].

It is a common belief that VOD is an effect rather than a cause of ED by medical professionals. VOD is attributable to an underlying pathologic process that is not generally correctable through surgery [32]. As a consequence, penile venous surgery in the management of ED is not well accepted by peers. The available evidence, up to now, does not support penile venous surgery as a treatment option for ED. In 2010, the ISSM consensus did not recommend penile venous surgery on a regular basis because of insufficient evidence to support the efficacy. Penile venous surgery is still considered investigational and it is recommended only in special situations, such as for young patients with site-specific congenital, post-traumatic, or post-inflammatory venous leaks. Similarly in 2014, the European Association of Urology (EAU) Guidelines on ED rejected penis venous ligation procedures as an option for ED management given the poor overall outcomes reported [33]. The sample size of PVE, PAR and PAS was too small to draw a conclusion in this study; however, we concur that the traditional PVL should not be recommended.

Objective vascular testing can provide a reliable physiologic diagnosis. We evaluated erectile hemodynamics chiefly by dual pharmacocavernosography with PGE1 injection to look for the presence of venous leakage, which is extremely informative for penile venous anatomy and physiology [34]. In our own experience, a radiopacity comparison was made between the CC especially the penile crura and the femoral cortex in all patients reported in this study. The radiopacity was remarkably enhanced by PVSS and this index is recommended to evaluate the adequacy of the surgery. Diagnostic dynamic penile duplex Doppler ultrasound (PDDU) is an alternative tool that can assess arterial function and the presence of significant cavernosal venous leakage by measuring peak arterial flow and the calculated resistive index [35]. Given the fact that surgery was performed on those who were undergoing multidisciplinary investigation elsewhere, this anatomy-based PVSS can be a viable option to prevent the CC from being VOD through firm ligation of the emissary veins at the closest vicinity to the outer layer of the tunica albuginea. Further research on the patho-physiology of ED is warranted because of the continued prevalence of the issue and the lack of reliable treatment options. 
In conclusion, our refined PVSS is found to be beneficial in most patients who underwent prior penile vascular interventions elsewhere. Due to the lack of reported experience, we would like to share our own findings to advocate for this evidencebased procedure that can benefit ED patients.

\section{Acknowledgement}

We would like to thank Director Chih-Chung Lu, Ms Hsiu-Chen Lu, Tzu-En Hsu and Venus Ying-Hui Chen for their preparations of the illustration and photos for this manuscript.

\section{REFERENCES}

Krane RJ, Goldstein I, Saenz de Tejada I. Impotence. N. Engl. J. Med. 321(24)16481659(1989).

Hsu GL, Hsieh CH, Wen HS, et al. Anatomy of the human penis: the relationship of the architecture between skeletal and smooth muscles. J. Androl. 25(3)426431(2004).

Gratzke C, Angulo J, Chitaley K, et al. Anatomy, physiology, and pathophysiology of erectile dysfunction. J. Sex Med. 7(1.2)44575(2010).

Hellstrom WJ, Montague DK, Moncada I, et al. Implants, mechanical devices, and vascular surgery for erectile dysfunction. J. Sex Med. 7,501-523(2010).

Michal V, Kramar R, Pospichal J, et al. Direct arterial anastomosis on corpora cavernosa penis in the therapy of erective impotence. Rozhl. Chir. 52(9)587590(1973).

Rogers JH, Goldstein I, Kandzari DE, et al. Zotarolimus-eluting peripheral stents for the treatment of erectile dysfunction in subjects with suboptimal response to phosphodiesterase-5 inhibitors. J. Am. Coll. Cardiol. 60(25)2618-2627(2012).

Cakan M, Yalcinkaya F, Demirel F, Ozgunay $\mathrm{T}$, Altug $U$. Is dorsale penile vein ligation (dpvl) still a treatment option in venoocclusive dysfunction? Int. Urol. Nephrol. 36(3)381-387(2004).

Hsu GL, Chen HS, Hsieh $\mathrm{CH}$, et al. Clinical experience of a refined penile venous stripping surgery procedure for patients with erectile dysfunction: is it a viable option? J. Androl. 31(3)271-280(2010).

Chocholatv M, Hanek P, Jarolim L, Padr R. Embolization of the deep dorsal vein using $96 \%$ ethanol for treatment of the venogenic erectile dysfunction. Eur. Urol. Suppl. 9, 643(2010).

Aschenbach R, Steiner T, Kerl MJ, et al. Endovascular embolisation therapy in men with erectile impotence due to veno-occlusive dysfunction. Eur. J. Radiol. 82(3)504-507(2013).

Kayigil O, Agras K, Okulu E. Is deep dorsal vein arterialization effective in elderly patients? Int. Urol. Nephrol. 40(1)125131(2008).
Chen SC, Hsieh $\mathrm{CH}$, Hsu GL, et al. The progression of the penile vein: could it be recurrent? J Androl. 26(1), 53-60 (2005).

Parona F. Imperfect penile erection due to varicosity of the dorsal vein: observation. Giornale Italiano Delle Malattie Veneree E. Della Pelle. 14, 71-76(1873).

Wooten JS. Ligation of the dorsal vein of the penis as a cure foratonic impotence. Texas Med. J. 18, 325-329(1902).

Lowsley OS, Rueda A. Further experience with an operation for the cure of certain types of impotence. J. Int. Coll Surg 19(1)6977(1953).

Wespes E, Schulman CC. Venous leakage: surgical treatment of a curable cause of impotence. J. Urol. 133(5)796-8(1985).

Montague DK, Barada JH, Belker AM, et al. Clinical guidelines panel on erectile dysfunction: summary report on the treatment of organic erectile dysfunction. The American Urological Association. J. Urol. 156(6)2007-2011(1996).

Berardinucci D, Morales A, Heaton JP, Fenemore J, Bloom S. Surgical treatment of penile veno-occlusive dysfunction: is it justified? Urology. 47(1)88-92(1996).

Hsu GL, Hsieh CH, Wen HS, et al. Penile venous anatomy: an additional description and its clinical implication. J. Androl. 24(6)921927(2003).

Wen $\mathrm{HS}$, Hsieh $\mathrm{CH}_{\text {, }} \mathrm{Hsu} \mathrm{GL}$, et al. The synergism of penile venous surgery and oral sildenafil in treating patients with erectile dysfunction. Int. J. Androl. 28, 297303(2005).

Anonymous. FDA approves oral therapy for erectile dysfunction. Am. J. Health Syst. Pharm. 55, 981-984(1998).

Montague DK. Penile prosthesis implantation in the era of medical treatment for erectile dysfunction. Urol. Clin. North Am. 38(2)217-225(2011).

Nehra A, Jackson G, Miner M, etal.The Princeton III Consensus recommendations for the management of erectile dysfunction and cardiovascular disease. Mayo Clin. Proc. 87(8)766-778(2012).

Liu H, Waite LJ, Shen S, Wang DH. Is Sex Good for Your Health? A National Study on Partnered Sexuality and Cardiovascular Risk among Older Men and Women. J.
Health Soc. Behav. 57(3)276-96(2016).

Elhanbly S, Abdel-Gaber S, Fathy $\mathrm{H}$, et al. Erectile dysfunction in smoker: a penile dynamic and vascular study. J. Androl. 25(6)991-995(2004).

Halliday D. Fluid Mechanics. Fundamentals of physics. 2nd Edition. Singapore: John Wiley \& Sons pp 297-308(1986).

Hsu GL, Chen HS, Hsieh CH, Lee WY, Chen KL and Chang $\mathrm{CH}$. Salvaging Penile Venous Stripping Surgery. J. Androl. 31(3)261262(2010).

Molodysky E, Liu SP, Hsu GL, Huang SJ. Penile vascular surgery for treatment of erectile dysfunction: Current role and future direction. Arab J. Urol. 11(3)254266(2013).

Hsieh $\mathrm{CH}$, Huang YP, Tsai $\mathrm{MH}$, et al. Tunical outer layer plays an essential role in penile veno-occlusive mechanism evidenced from electrocautery effects to the corpora cavernosa in defrosted human cadavers. Urology. 86(6)1129-1135(2015).

Hsu GL, Hung YP, Tsai MH, et al. Penile veins are the principal component in erectile rigidity: a study of penile venous stripping on defrosted human cadavers. J. Androl. 33(6)1176-1185(2012).

Kayigil O, Okulu E, Aldemir M, et al. Penile revascularization in vasculogenic erectile dysfunction (ED): long-term follow-up. BJU Int. 109(1)109-115(2012).

Sohn M, Hatzinger M, Goldstein I, Krishnamurti S. Standard operating procedures for vascular surgery in erectile dysfunction: revascularization and venous procedures. J. Sex Med. 10, 172-179(2013).

Hatzimouratidis K, Eardley I, Giuliano F, et al. Guidelines on male sexual dysfunction: erectile dysfunction and premature ejaculation. European Association of Urology. http://uroweb.org/wp-content/ uploads/14-Male-Sexual-Dysfunction_ LR.pdf. (2014)

Hsu GL, Hill JW, Chen HS, Huang SJ. Novel pilot films providing a dispensable information in pharmaco-cavernosography. Transl. Androl. Urol. 4,398-405(2014).

Aversa A, Sarteschi LM. The role of penile colorduplex ultrasound for the evaluation of erectile dysfunction. J. Sex Med. 4(5)14371447(2007). 